Research Article

\title{
Numerical and Experimental Study on Soil Plug Resistance of Open-Ended Pipe Pile with a Restriction Plate
}

\author{
Yuan Guo $\mathbb{D}^{1},{ }^{1}$ Shaoyang Dong, ${ }^{2}$ Jiale $\mathrm{Li}^{3}$ and Xiong (Bill) Yu ${ }^{2}{ }^{2}$ \\ ${ }^{1}$ School of Civil Engineering, Sun Yat-Sen University, Guangzhou 510275, China \\ ${ }^{2}$ Department of Civil Engineering, Case Western Reserve University, Cleveland, OH 44106, USA \\ ${ }^{3}$ School of Civil and Transportation Engineering, Hebei University of Technology, Tianjin 300401, China \\ Correspondence should be addressed to Yuan Guo; yguo4@clemson.edu and Xiong (Bill) Yu; xxy21@case.edu
}

Received 30 October 2019; Accepted 7 May 2020; Published 10 June 2020

Academic Editor: Xueping Fan

Copyright (C) 2020 Yuan Guo et al. This is an open access article distributed under the Creative Commons Attribution License, which permits unrestricted use, distribution, and reproduction in any medium, provided the original work is properly cited.

The end bearing capacity of open-ended pipe piles is highly dependent upon the soil plugging behavior inside the pipe wall. For largediameter open-ended pipe piles, the arching effect due to inner soil-pile friction may be insufficient to generate a fully plugged condition, which compromises the end bearing capacity after the pile installation. Here, we propose two innovatively designed restriction plates installed inside the pipe to facilitate the soil plugging process, that is, a restriction plate with one circular hole and a restriction plate with four semisized circular holes. By use of the discrete element method, the mechanical behaviors of soil plugs in cohesionless soils with different restriction plates are analyzed. The numerical model has been validated by comparing the simulations to the results of a series of laboratory-scale experiments over different pile diameters, plug length-diameter ratios, and different types of restriction plates. It is shown that the numerical model can accurately predict the soil plug resistance and the particle-scale force transmission. Both numerical simulations and experiments validate that the arching effect is significantly enhanced by the restriction plate, especially with the four-hole restriction plate. Furthermore, the geotechnical centrifuge principle is adopted into the DEM model to study the mechanical behavior of large-diameter soil plugs. It is found that the effectiveness of restriction plate decreases as the pile diameter increases. However, even for the $2 \mathrm{~m}$ in diameter soil plugs, the plug resistance is increased by $252 \%$ with one-hole restriction plate and $281 \%$ with four-hole restriction plate compared to that of traditional pipe piles.

\section{Introduction}

Large-diameter open-ended pipe piles (LDOEPPs) typically refer to steel pipe piles with diameters larger than $0.91 \mathrm{~m}$ (or 36 inches) [1]. While the initial uses and experience with LDOEPPs are accumulated in the offshore industry $[2,3]$, the transportation community found them advantageous in providing large axial and lateral bearing capacities. Mohan et al. [4] have reported the use of LDOEPPs with diameters of $1.8 \mathrm{~m}$ to $2.5 \mathrm{~m}$ for the San Francisco-Oakland bay bridge due to their superior compression and tension capacities. As the pile can be driven installed in water, there is no need to construct a cofferdam as is needed for the construction of drilled shafts. The savings in construction cost and the reduction of construction schedule can be substantial. An example application in California showed that the use of
LDOEPPs for a bridge project reduced the construction time by half and led to a cost saving of $\$ 2.8$ million [5].

Despite the advantages in the transportation engineering, there are also challenges in the design and construction of LDOEPPs. The bearing capacity and drivability of openended pipe piles depend largely on the degree of soil plugging [6]. As the pile driven into the ground, soils are squeezed inside the pipe and form the arching effect in the soil plug, which significantly increases the inner unit area frictional resistance compared to that on the outer surface [7]. Laboratory experiments using photoelastic materials demonstrate the concentrated force transmission and arching effect at the bottom of soil plug [8]. Under static loading condition, there is a significantly high load transfer over the bottom soil with a height of three to four times of pile diameter [9]. Field tests on large-diameter pipe piles 
show that a relatively large displacement is required in order to fully mobilize the plug resistance [10]. It is also shown that the arching effect inside soil plug decreases as the diameter of pipe pile increases [11]. These introduce large uncertainties in the design and construction of LDOEPPs, especially in the estimation of end bearing capacity and drivability.

The recommended design practice of the American Petroleum Institute (API) describes the total axial resistance of LDOEPPs as the sum of the external shaft friction and the end bearing on the pipe wall annulus plus the plug resistance or the end bearing of the pile over the total cross section, whichever is the lesser, as shown in equation (1) [12, 13]. There are various factors determining the plugging behavior: the pile diameter, length-diameter ratio, installation method (driven or jacked pile), soil mechanical property, and friction at soil-pile interface [14-16]. A strength reduction of soil plug has been observed under dynamic loading conditions $[17,18]$. Experiments also show that the degree of soil plug increases with the increase of the relative density of sands [19]:

$$
Q_{c}=Q_{f}+\min \left\{Q_{\mathrm{ann}}+Q_{p}, Q_{\text {tip }}\right\},
$$

where $Q_{c}$ is the total axial resistance of the open-ended pipe pile; $Q_{f}$ is the external shaft friction; $Q_{a n n}$ is the resistance of pipe wall annulus; $Q_{p}$ is the plug resistance; and $Q_{\text {tip }}$ is the tip resistance over the total cross section area.

The soil plugging performance can also be estimated based on pile dimensions as well as soil mechanical strength, which can be acquired from the Cone Penetration Test (CPT) or the standard penetration test [20-24]. Some typical design methods include the CPT-based University of Western Australia method [25] and the Imperial College Pile method [26]. Besides, some in situ parameters, such as the soil plug incremental filling ratio, which equals the soil plug length increment divided by the pile driving increment, have been incorporated into the design practice to evaluate the degree of soil plugging $[27,28]$. However, the field tests show that the CPT-based method provides reliable prediction on skin friction, while the estimation of end bearing capacity is still challenging [29].

Researchers have proposed different mathematical models to study the bearing capacity and settlement behavior of open-ended pipe piles under various loading conditions [30-33]. Numerical methods, for example, finite element method and coupled Eulerian-Lagrangian numerical technique, are used to study the mechanical behavior of LDOEPPs [34, 35]. The discrete element method (DEM) proposed by Cundall and Strack [36] is found advantageous, especially in the simulation of soil arching inside the pipe pile $[37,38]$. This paper aims to discuss the effectiveness of two innovatively designed restriction plates that welded inside the pipe pile, that is, a donut-shaped one-hole restriction plate (see Figure 1) and a semisized four-hole restriction plate with the same opening plate area. A DEM model has been built to study the force transmission inside the soil plug with different restriction plates. The numerical model has been validated by comparison to a series of laboratory soil plug loading experiments to study the soil plug resistance over different pile diameters, plug lengthdiameter ratios, and different types of restriction plates.

\section{Numerical Method and Model Setup}

The discrete element method is able to simulate the soil plug formation and the arching effect at the grain scale. By solving the motion of individual particles, the DEM model readily describes the particle-scale interaction and particle rearrangement inside the soil skeleton, which is challenging for the continuum-based numerical methods.

2.1. Mathematical Equations. In the DEM model, spherical particles are subjected to the body force and the contact force from interacting objects and are governed by Newton's laws of motion [36]. The conservations of linear momentum and angular momentum for each particle follow equations (2) and (3). The soil particles are assumed in a dry condition for simplicity; as a result, the buoyant force and pore water pressure have been neglected in this study:

$$
\begin{aligned}
& m_{i} \frac{\mathrm{d} u_{i}}{\mathrm{~d} t}=\sum_{j=1}^{n_{i}} F_{c, i j}+F_{g, i}, \\
& I_{i} \frac{\mathrm{d} w_{i}}{\mathrm{~d} t}=\sum_{j=1}^{n_{i}} M_{i j},
\end{aligned}
$$

where $m_{i}$ is the particle mass; $u_{i}$ is the translational velocity; $t$ is the time; $n_{i}$ is the total number of contacts; $F_{c, i j}$ is the contact force between two particles; $F_{g, i}$ is the particle gravitational force, and $F_{g, i}=4 / 3 \pi R_{i}^{3} \rho_{i} g ; R_{i}$ is the particle radius; $\rho_{i}$ is the density; $g$ is the gravitational acceleration; $I_{i}$ is the particle moment of inertia; $w_{i}$ is the angular velocity; $M_{i j}$ is the torque between two particles; and $i$ and $j$ indicate associated particles.

The rheological components of the contact model are shown in Figure 2. There are a linear spring and a dashpot in the normal and tangential directions and a slider in the tangential direction of each contact. The viscous damping model is used to dissipate particle's kinetic energy, and there is no local damping applied in the simulation. The mathematical definition of the contact stiffness model is given in equations (4) and (5) [39]. The slip model allows slides to occur by limiting the tangential contact force to a maximum shear force (see equation 6):

$$
\begin{gathered}
F_{c, i j}^{n}=k_{i j}^{n} \delta_{i j}^{n}, \\
\Delta F_{c, i j}^{s}=-k_{i j}^{s} \Delta \delta_{i j}^{s}, \\
\left|F_{c, i j}^{s}\right| \leq \mu\left|F_{c, i j}^{n}\right|,
\end{gathered}
$$

where $F_{c, i j}^{n}$ is the normal contact force; $k_{i j}^{n}$ is the normal secant stiffness; $\delta_{i j}^{n}$ is the normal relative displacement; $\Delta F_{c, i j}^{s}$ is the increment of shear contact force; $k_{i j}^{s}$ is the shear tangent stiffness; $\Delta \delta_{i j}^{s}$ is the increment of shear relative displacement; $\mu$ is the friction coefficient; and $i$ and $j$ indicate associated particles. 


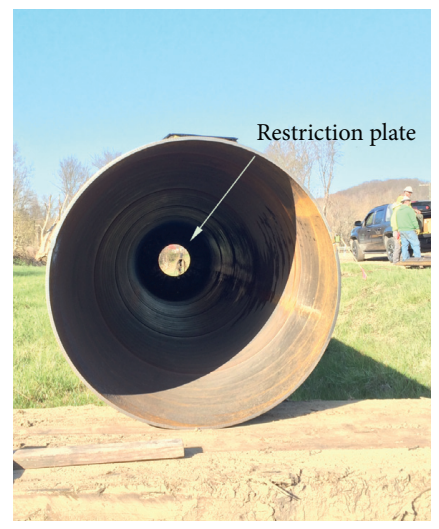

(a)

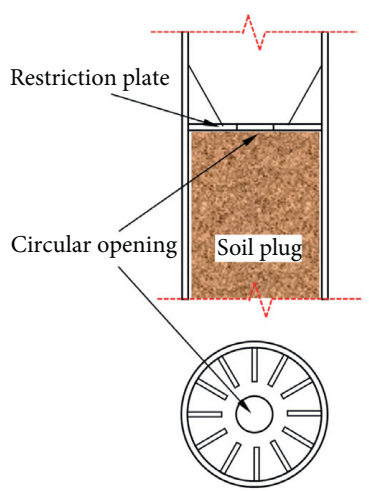

(b)

FIGURE 1: Large-diameter open-ended pipe pile with a donut-shaped restriction plate in New Philadelphia, Ohio.

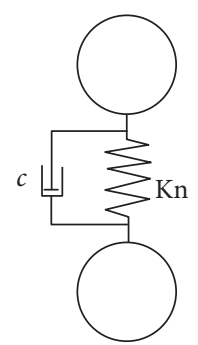

(a)

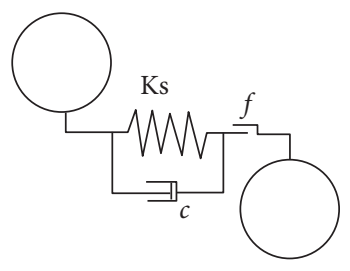

(b)

FIGURE 2: Rheological components of the force-displacement law between two particles.

2.2. Description of Numerical Model. A laboratory-scale soil plug model is built to analyze the soil plugging behavior inside the open-ended pipe pile, as illustrated in Figure 3. The mechanical performance of the external shaft friction of open-ended pipe piles is generally similar to that of closedended pipe piles. As a result, only the soil plug portion is considered in this model to optimize the computational cost. Experiments conducted by O'Neill and Raines [9] showed that there was a high load transfer at the bottom of soil plug with a height of about three to four times of the pipe diameter. Based on this observation, a soil plug model with a length-diameter ratio $(L / D)$ of 4.0 is built to study the frictional resistance and the arching effect. A detailed discussion on the length-diameter ratio is provided in the following section. The diameter of the pipe is set to $10 \mathrm{~cm}$ and the height of soil plug is $40 \mathrm{~cm}$ to ensure efficient computational solutions.

The model is generated by depositing the soil particles inside the pipe and settling under gravity to simulate the air pluviation in preparing cohesionless soil samples. Since the objective of this model is to study the plug resistance, the bottom wall is defined as a fixed boundary, so that as the pipe wall moves downwards, the soil plug resistance can be fully mobilized. Only spherical particles are used to emulate the granular soil grains, and the particle angularity is considered implicitly by calibrating the internal friction angle. The size of DEM particles follows a uniform distribution ranging

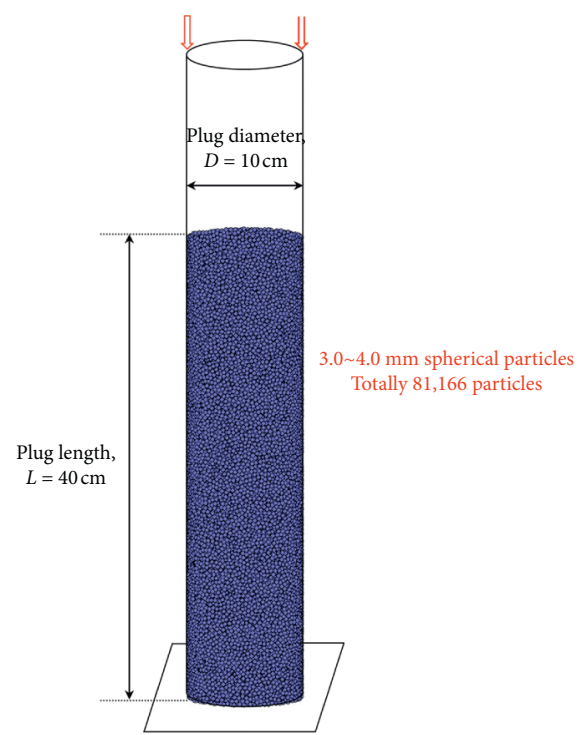

FIgUre 3: Illustration of the DEM model of soil plug.

from $3.0 \mathrm{~mm}$ to $4.0 \mathrm{~mm}$. In total, there are 81,166 spherical particles generated in the DEM model.

The particle contact parameters are calibrated by conducting the triaxial test simulations and comparing Young's modulus and internal friction angle to the results measured from the experiments. The normal and shear particle contact 
stiffnesses are assumed to be the same to simplify the calibration. Young's modulus is used to determine the contact stiffness of particles, and the internal friction angle is used to determine the particle friction coefficient. The particle density is calibrated by the experimentally measured bulk density of sand samples prepared by the air pluviation method. Table 1 summarizes the calibrated particle contact parameters used in the following simulations. These particle parameters characterize the soil sample with a Young's modulus of $8.57 \mathrm{MPa}$ under $200 \mathrm{kPa}$ confining pressure and an internal friction angle of $28.6^{\circ}$. The friction coefficient of soil-pipe interface is measured from the direct shear test on sand grains shearing on a steel plate, which provides a friction coefficient of 0.33 .

2.3. Particle Size and Pile Moving Velocity. The grain size and the pile moving velocity are two important factors that determine the simulated soil plug resistance. A sensitivity study has been conducted to analyze their influence on soil plug's mechanical behavior. Typically, in the DEM models, the size of particles is generally much larger than the size of realistic soil grains due to the limited computational resource. For a given sized numerical model, the number of particles increases cubically with the decrease of particle size. Figure 4(a) compares the soil plug resistance and the total number of particles from simulations over different mean particle sizes (from $2.5 \mathrm{~mm}$ to $6.5 \mathrm{~mm}$ ). The diameter of the pipe pile is $10 \mathrm{~cm}$ and the length-diameter ratio is 2.0 . The plug resistance is defined as the maximum frictional resistance on the cylindrical wall while driving, typically reached within a displacement of $25 \%$ of the pile diameter. It can be seen from Figure 4(a) that the plug resistance increases slightly with the increase of particle size; however, the number of particles used to generate the DEM model decreases dramatically. Considering the little variation in plug resistance over different particle diameters, a particle size range of $3.0 \mathrm{~mm}$ to $4.0 \mathrm{~mm}$ is chosen in the following simulations.

Figure 4(b) shows the results of a sensitivity study on pile's moving velocity. In this simulation, pipe piles are assumed to move downwards with constant velocities. In the field installations, pipe piles are typically driven by impact loads, and the associated soil disturbance may also influence the plugging performance, which is not considered in this study for simplicity. From Figure 4(b), as the pile's moving velocity increases from $0.1 \mathrm{~mm} / \mathrm{s}$ to $100 \mathrm{~mm} / \mathrm{s}$, the plug resistance increases from $245 \mathrm{~N}$ to $1,205 \mathrm{~N}$. A significant increment occurs as the pile moving velocity exceeds $3.0 \mathrm{~mm} / \mathrm{s}$. For models with moving velocity smaller than $1.0 \mathrm{~mm} / \mathrm{s}$, the plug resistances are similar. Regarding these observations, the pile moving velocity is set to $1.0 \mathrm{~mm} / \mathrm{s}$ for both simulations and laboratory experiments to eliminate the additional influence from the pile moving.

\section{Model Validation by Experimental Tests}

In conjunction with the numerical simulations, a series of laboratory experiments have been conducted to study the
TABle 1: Particle parameters used in the DEM model.

\begin{tabular}{lc}
\hline Parameter & Value \\
\hline Particle size & $3.0 \sim 4.0 \mathrm{~mm}$ \\
Particle density & $2683 \mathrm{~kg} / \mathrm{m}^{3}$ \\
Initial porosity & 0.42 \\
Normal/shear contact stiffness & $4.5 \times 10^{5} \mathrm{~N} / \mathrm{m}$ \\
Particle friction coefficient & 0.65 \\
Critical damping ratio & 0.1 \\
Wall contact stiffness & $1.0 \times 10^{8} \mathrm{~N} / \mathrm{m}$ \\
Soil-steel friction coefficient & 0.33 \\
Characterized friction angle & $28.6^{\circ}$ \\
Characterized Young's modulus & $8.57 \mathrm{MPa}$ \\
\hline
\end{tabular}

mechanical behavior of soil plugs under different pile diameters, plug length-diameter ratios, and restriction plates. The experimental results also serve to validate the numerical model in estimating the soil plug resistance.

3.1. Experimental Setup. Figure 5 illustrates the experimental setup of the soil plug resistance test, which follows the same setup of the numerical model. The sand sample is provided by a local commercial supplier in Northeast Ohio. From the sieve analysis, the mean particle size is $0.20 \mathrm{~mm}$ with a uniformity coefficient of 2.10 and a coefficient of gradation of 1.22. According to the unified soil classification system, the sand sample is classified as the poor graded sand (SP). The internal friction angle is $29.1^{\circ}$, measured from a series of direct shear tests with loose samples prepared by the air pluviation method. The maximum/minimum dry density and the specific gravity of sand particles are also measured following the standards [40-42]. Table 2 summarizes the mechanical properties of the sand sample characterized from the laboratory experiments.

The soil plug specimen is prepared inside the pipe pile by the air pluviation method, where sand samples are poured from the top of the pipe and settled to form a loose state. The bottom of the soil plug is supported by a fixed flat base strut. A layer of grease has been applied to seal the gap between the base strut and the pipe wall, which also helps to minimize the friction possibly generated between the base strut and the pipe wall. The measured sand density after the deposition is $1.52 \mathrm{~g} / \mathrm{cm}^{3}$. Once prepared, a vertical force is applied to the pipe pile to drive it downwards with a constant velocity of $1.0 \mathrm{~mm} / \mathrm{s}$ by a loading piston mounted on the loading frame. The loading frame has a maximum limit of $25,000 \mathrm{~N}$ for the axial loading force considering the possible buckling. The vertical displacement and the axial force are recorded during the test.

Two innovatively designed restriction plates are proposed to enhance the soil plugging effect for the large-diameter pipe piles, which are generally difficult to form a fully plugged state due to the small inner friction. The two types of restriction plates are one-hole restriction plate and four-hole restriction plate. The geometric dimensions of restriction plates are shown in Figure 6(a). For the one-hole restriction plate, the inner diameter of circular hole is one half of the pipe diameter, similar to the field design (see Figure 1). For the four-hole restriction plate, the diameter of circular holes 


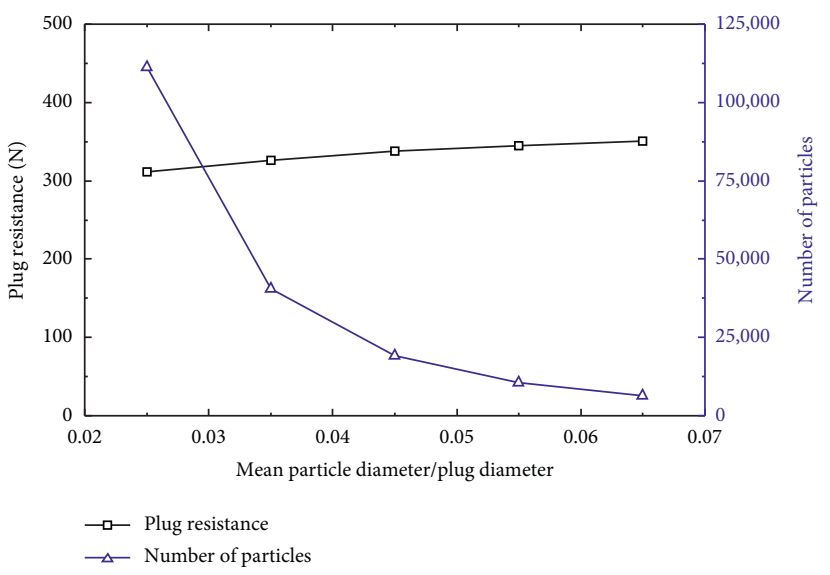

(a)

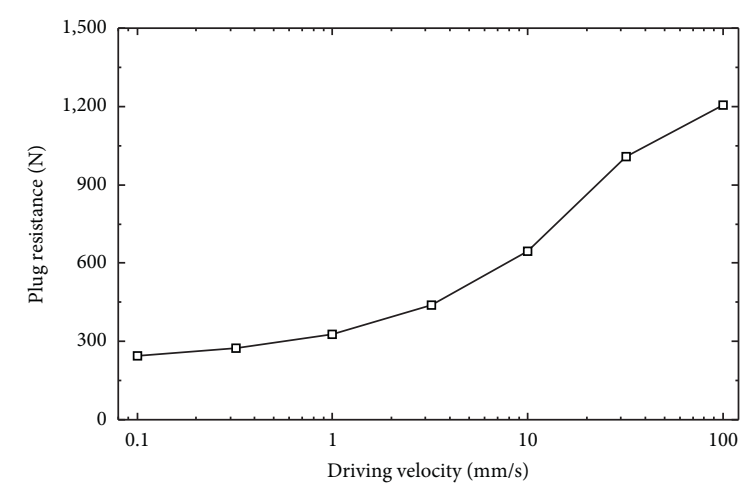

(b)

FIGURE 4: Sensitivity study of the soil plug model on (a) particle size and (b) wall moving velocity $(D=10 \mathrm{~cm}$ and $L / D=4.0)$.
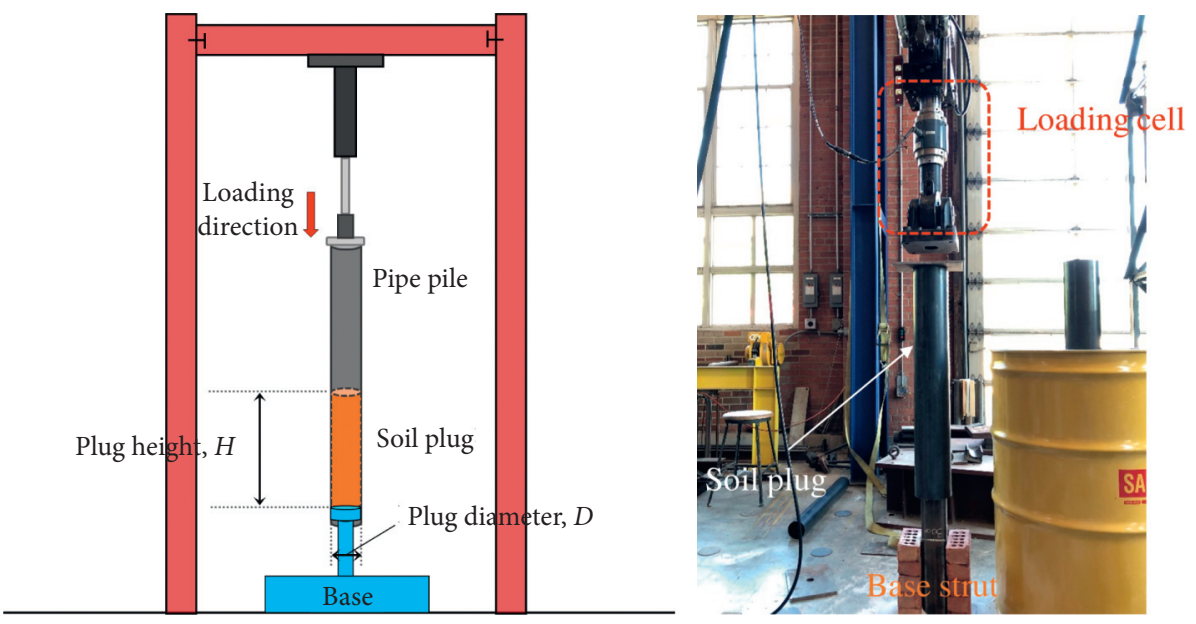

Figure 5: Experimental setup of the soil plug resistance test.

TABLE 2: Mechanical properties of sand samples used in laboratory experiment.

\begin{tabular}{lc}
\hline Property & Value \\
\hline Mean grain size & $0.20 \mathrm{~mm}$ \\
Uniformity coefficient, $C_{\mathrm{u}}$ & 2.10 \\
Coefficient of gradation, $C_{\mathrm{c}}$ & 1.22 \\
Sand specific gravity & $2683 \mathrm{~kg} / \mathrm{m}^{3}$ \\
Maximum-index dry density & $1.67 \mathrm{~g} / \mathrm{cm}^{3}$ \\
Minimum-index dry density & $1.43 \mathrm{~g} / \mathrm{cm}^{3}$ \\
Maximum void ratio & 0.876 \\
Minimum void ratio & 0.607 \\
Internal friction angle & $29.1^{\circ}$ \\
\hline
\end{tabular}

is one-fourth of the pipe diameter, so that the total effective plate areas of the two types of plates are the same. Three different-sized pipe piles have been used for the soil plug test with the pile diameters of $5.0 \mathrm{~cm}, 10.0 \mathrm{~cm}$, and $15.0 \mathrm{~cm}$, and the length of the piles is $1.0 \mathrm{~m}$. The heights of soil plugs are prepared following different length-diameter ratios, that is, 2.0, 4.0, 6.0, and 8.0. A trial test on the $5.0 \mathrm{~cm}$ in diameter soil plug with an $L / D$ of 10.0 showed that the axial force has exceeded the loading frame's limit of $25,000 \mathrm{~N}$.
Table 3 summarizes the model configurations for the loading experiments and the obtained soil plug resistance. The plug resistance is defined as the peak axial resistance within the displacement of $25 \%$ of the pile diameter, as most of the peak strength occurs within this range. There are ten cases tested with different pile diameters, length-diameter ratios, and types of restriction plates. Figure 6(b) provides the top views of the pipe piles before and after driving, corresponding to the cases of \#6, \#9, and \#10 (see Table 3). As can be seen, soil columns are squeezed out from the top holes under the axial load, and the four-hole restriction plate provides a higher confinement in terms of the amount of soils extruded compared to the one-hole restriction plate.

3.2. Plug Length-Diameter Ratio. The corresponding DEM simulations are also performed and compared to the experimental results to demonstrate the model capabilities. The particle contact parameters of the DEM model remain the same as listed in Table 1. The soil plug length-diameter ratio is an important factor determining the degree of soil plugging inside the LDOEPPs. Similar to the experimental 

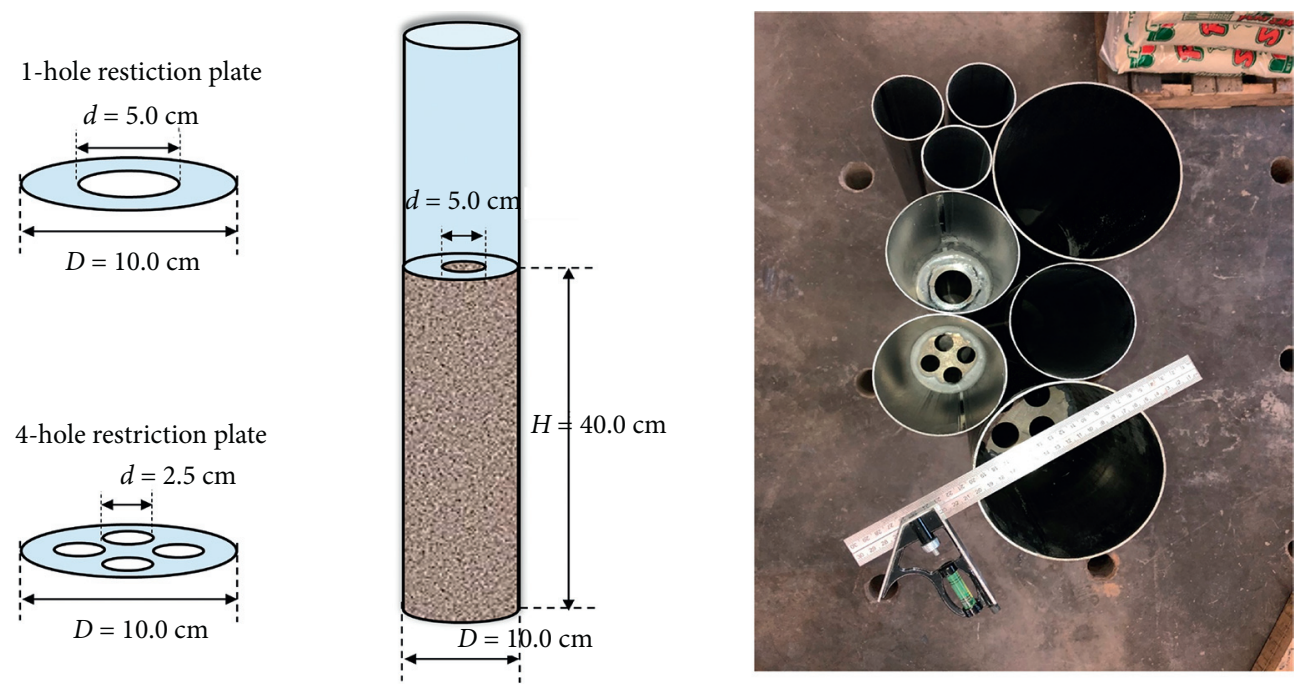

(a)
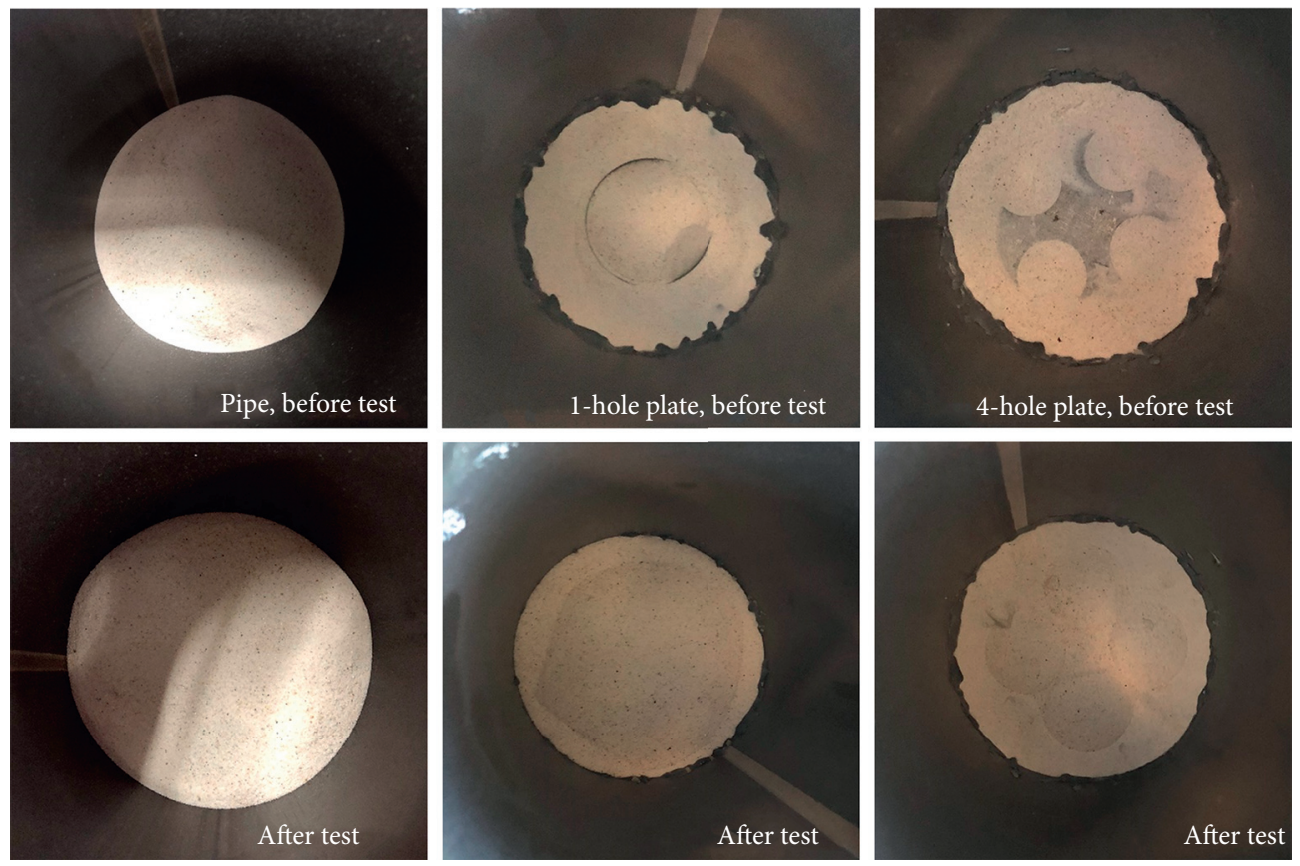

(b)

FIGURE 6: Soil plug resistance test with different restriction plates: (a) model dimensions and (b) top view of soil plug before and after the test.

TABLE 3: Geometric dimensions of soil plug models used in laboratory experiment.

\begin{tabular}{lcccc}
\hline$\#$ & Pile diameter $(\mathrm{cm})$ & Length-diameter ratio & Restriction plate & Plug resistance \\
\hline 1 & 5 & 2.0 & N.A. & 322.7 \\
2 & 5 & 4.0 & N.A. & 743.1 \\
3 & 5 & 6.0 & N.A. & $3,013.1$ \\
4 & 5 & 8.0 & N.A. & $7,514.2$ \\
5 & 10 & 2.0 & N.A. & 183.4 \\
6 & 10 & 4.0 & N.A. & $2,059.2$ \\
7 & 15 & 2.0 & N.A. & 173.7 \\
8 & 15 & 4.0 & N.A. & $1,350.6$ \\
9 & 10 & 4.0 & One-hole plate & $>25,000 \mathrm{~N}$ \\
10 & 10 & 4.0 & Four-hole plate & $>25,000 \mathrm{~N}$ \\
\hline
\end{tabular}


program, the pipe with a diameter of $5.0 \mathrm{~cm}$ is chosen to build the numerical models with different $L / D$ s. Figure 7 (a) compares the load-displacement curves between experiments and simulations with $L / D$ s of 6.0 and 8.0. From Figure $7(\mathrm{a})$, it can be seen that a large pile displacement (around 20\% of pile diameter) is required to fully mobilize the peak resistance, especially for the numerical models.

Figure 7(b) compares the soil plug resistance obtained from numerical and experimental tests over different plug lengthdiameter ratios. The numerical model predicts the experimental result surprisingly well by use of the calibrated particle contact parameters as discussed above. As shown in Figure 7(b), the plug resistance increases about cubically with the increase of $L$ / $D$, indicating a dramatic change of the degree of soil plugging. However, it should be noted that, for a fully plugged pipe pile, the plug resistance can be extremely large, and the equivalent tip resistance is determined by the end bearing capacity over the whole pile tip (see equation (1)). In that case, the open-ended pipe pile will behave as a closed-ended pipe pile.

3.3. Pile Diameter. The soil plug resistance is also determined by the pile diameter, as the small pipe piles are easier to be plugged than the large pipe piles with the same plug length-diameter ratio. Similar to the experiments, three different pipe pile models are generated with pile diameters of $5.0 \mathrm{~cm}, 10.0 \mathrm{~cm}$, and $15.0 \mathrm{~cm}$ and plug length-diameter ratios of 2.0 and 4.0. Figure 8 compares the plug's unit area resistance from the experiments and simulations over different pile diameters. The plug unit area resistance is defined as the plug resistance divided by the cross section area of the pile. Generally, a larger unit area resistance indicates a higher degree of soil plugging. From the experimental result, the unit area resistance decreases with the increase of pile diameter, indicating a decrease of degree of soil plugging, that is, the unit area resistance decreases from $379 \mathrm{kPa}$ to $76 \mathrm{kPa}$ as the pipe pile diameter increases from $5.0 \mathrm{~cm}$ to $15.0 \mathrm{~cm}$. Both the experiment and the simulation indicate that the mechanical behavior of large-diameter pipes is quite different from that of small-diameter pipes.

It is also observed that there is a relatively large deviation between the simulation and the experiment for the $5.0 \mathrm{~cm}$ in diameter pipe models. This possibly results from the simplified spherical particle shape compared to the realistic sand grains. In the DEM model, sand grain's angularity is implicitly considered by the proper selection of frictional parameters. This works well in terms of the stress-strain behavior of granular soils in the normal stress regime. However, the influence of grain shape on soil's volumetric strain is generally ignored. For the sand samples used in the experiment, the void ratio varies from 0.607 to 0.876 , while in the numerical model built with spherical particles, the varying range is much smaller (the final void ratio after deposition is around 0.42 ). The physical model features a loose packing state due to the irregular grain shape and will be compacted by particle rearrangement, especially under high confining pressures as is the case for small-diameter piles. The current numerical model may not properly consider the evolution of soil's microfabric.
3.4. Restriction Plate. From the experimental tests, the implementation of restriction plate significantly improves the soil plug's frictional resistance: the plug resistance of the $10.0 \mathrm{~cm}$ in diameter soil plug is $2,059 \mathrm{~N}$ without a restriction plate, while with the installation of the restriction plate, the plug resistance exceeded the upper limit of the loading frame of $25,000 \mathrm{~N}$. The numerical model also predicts a remarkable improvement on soil plug's frictional resistance. Figure 9 compares the experimental and numerical force-displacement curves of $10.0 \mathrm{~cm}$ in diameter soil plugs with different restriction plates. Both the numerical model and physical model show that the plug resistance increases by over ten times by incorporating the restriction plate. The soil plug with four-hole plate tends to be stiffer than that with onehole plate, which is consistent with the observations on soil extrusion after the test (Figure 6(b)). Generally, the fourhole restriction plate provides a higher degree of plugging due to the higher arching effect with small holes compared to one-hole restriction plate.

Besides, the load-displacement behaviors of the numerical soil plug model with a restriction plate feature a "strain-softening" behavior (Figure 9(a)), which means pipe piles with restriction plates need a longer driving distance to fully mobilize the plug resistance. The possible reason is that the linear contact model adopted may not fully describe the physical contacts between sand particles, especially under high confining pressures. The particle contact stiffness was calibrated by Young's modulus from the triaxial test with a confining pressure of $200 \mathrm{kPa}$, while the confinement inside a plugged soil column may be well beyond this range. Other nonlinear contact models may help to better improve the model performance. Overall, the proposed DEM model captures the mechanical behaviors of soil plugs of different length-diameter ratios, pile diameters, and different types of restriction plates with a reasonable accuracy.

\section{Further Discussion}

The formation of the soil plug is further analyzed by looking into its particle-scale characteristics as well as the force transmission inside the soil plug with different restriction plates. Moreover, by adopting the geotechnical centrifuge principle, the numerical model is scaled up to analyze the soil plug resistance of large sizes.

4.1. Particle-Scale Characteristics. The formation of the soil plug is highly associated with the localization of interparticle contacts, which can be easily visualized by the DEM model. Figure 10 compares the contact force transmission inside a $10 \mathrm{~cm}$ in diameter soil plug in the pile driving process. As can be seen, soil particles are first balanced under gravity and the maximum contact force on individual particles is only $0.2 \mathrm{~N}$. The particle contact force increases dramatically as the pipe wall moves downward. At a driving displacement of $10.0 \mathrm{~mm}$ (or $10 \%$ of the pile diameter), the maximum normal contact force reaches $9.7 \mathrm{~N}$. As the pipe drives further to $20.0 \mathrm{~mm}$ (or $20 \%$ of the pile diameter), the maximum normal contact force decreases to $6.7 \mathrm{~N}$ due to the 


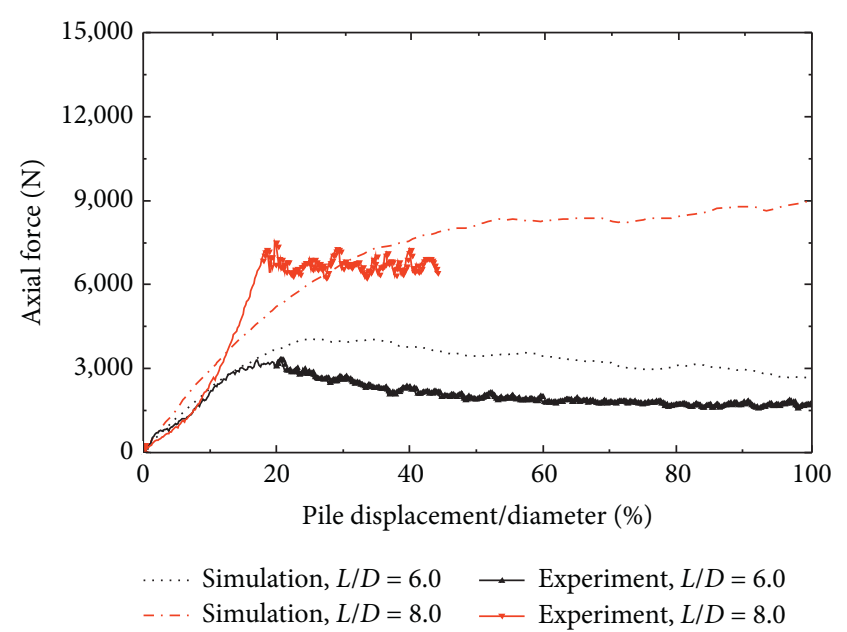

(a)

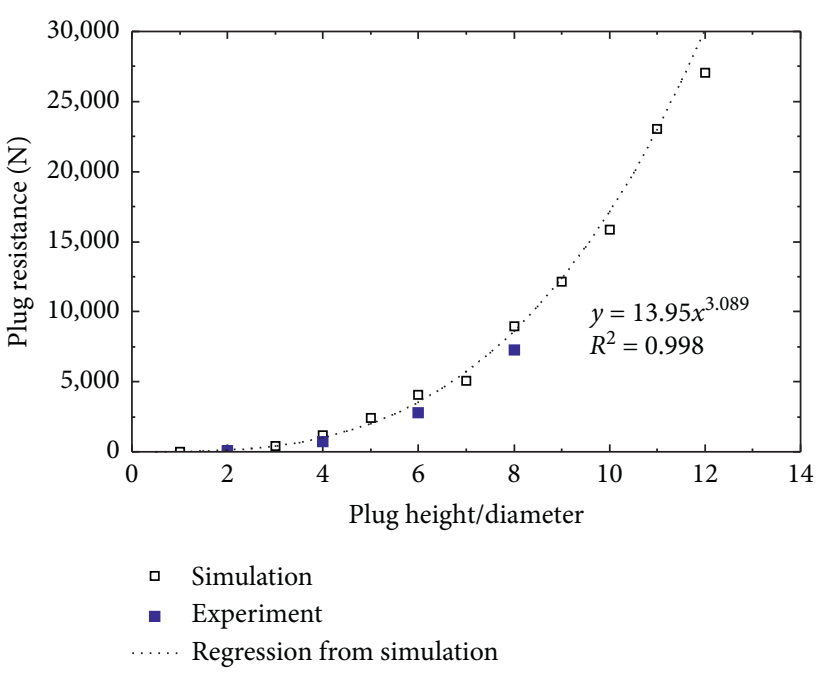

(b)

Figure 7: Experimental and simulation results of pipe piles with different plug length-diameter ratios: (a) examples of force-displacement curves and (b) plug resistance over different $L / D \mathrm{~s}(D=5.0 \mathrm{~cm})$.

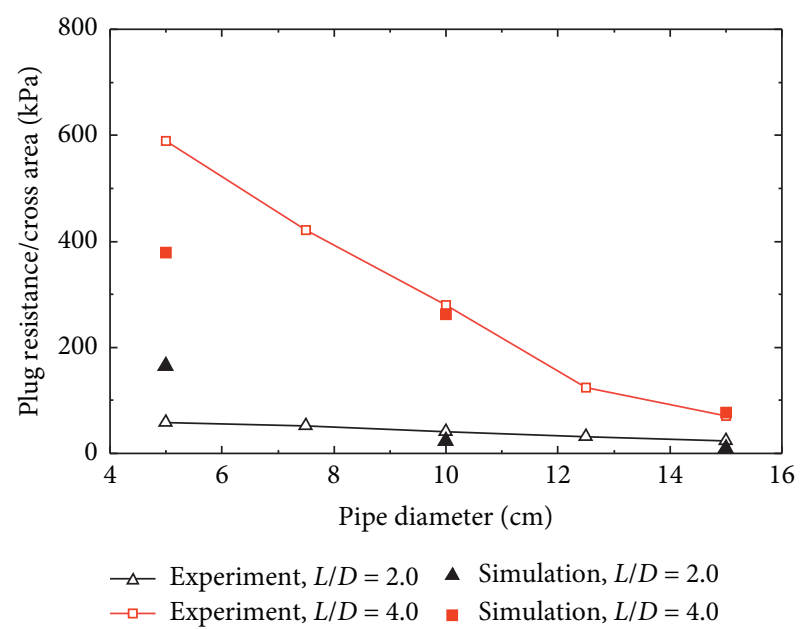

Figure 8: Comparison between experiments and simulations on soil plug resistance over different pile diameters.

particle relocation and stress release while shearing. It is also observed that the particle contact force concentrates at the bottom of the soil plug, while the upper portion experiences a relatively small compaction, which is consistent with the documented experiment conducted by use of photoelastic materials [8].

The porosity distribution inside the soil column is shown in Figure 11. The soil porosity at the specific location is approximated using a $10 \mathrm{~mm}$ in diameter measuring sphere, in which the porosity is calculated as the ratio of the voidspace volume inside the measuring sphere to the total volume of the measuring sphere. As the pile moves downward, the porosity at the bottom of the soil plug decreases due to compaction. It is also shown that the porosity at the soil-pipe interface increases while driving, indicating the development of shear band near the soil-structure interface.
As the formation of the soil plug results from the transmission of friction on the soil-pile interface, the friction coefficient at the soil-pile interface is expected to be a determinant factor on soil plug's performance. A sensitivity study on the soil-pile friction coefficient has been conducted and the results are shown in Figure 12. The pile diameter is $10.0 \mathrm{~cm}$ and the height of soil plug is $40.0 \mathrm{~cm}$. A sharp increase in plug resistance is observed as the soil-pipe friction coefficient increases from 0.26 to 0.53 . This may provide implications that the proper treatment on the pipe's inner surface may significantly change the pile drivability and mechanical behavior of the open-ended pipe piles.

4.2. Force Transmission with Restriction Plate. With the implementation of restriction plates, the transmission path of particle contact forces changes as well. Figure 13 compares the contact force distribution inside the $10 \mathrm{~cm}$ in diameter soil plugs with different types of restriction plates. The plug length-diameter ratio is set to 4.0. Three cases are simulated for the one-hole restriction plates with inner circular holes of $0.75 D, 0.50 D$, and $0.25 D$, where $D$ is the pile diameter. With the installation of restriction plates, soil particles on the top of the soil plug form the arching effect as well. Soil particles below the semicircle-shaped arching are subjected to much larger interparticle forces. This arching effect formed at the restriction plate prohibits the further movement of soil particles at the bottom of soil plug, which significantly improves the soil plug resistance. For the one-hole restriction plates, the arching effect increases with the decrease of the inner hole diameter.

Figure 14 compares the calculated particle stress along the soil plug length at the driving distance of $10 \mathrm{~mm}$. The particle contact stress is defined as the maximum contact force divided by particle's cross section area, as shown in equation (7). It should be noted that this definition is different from the stress concept in the continuous mechanics 


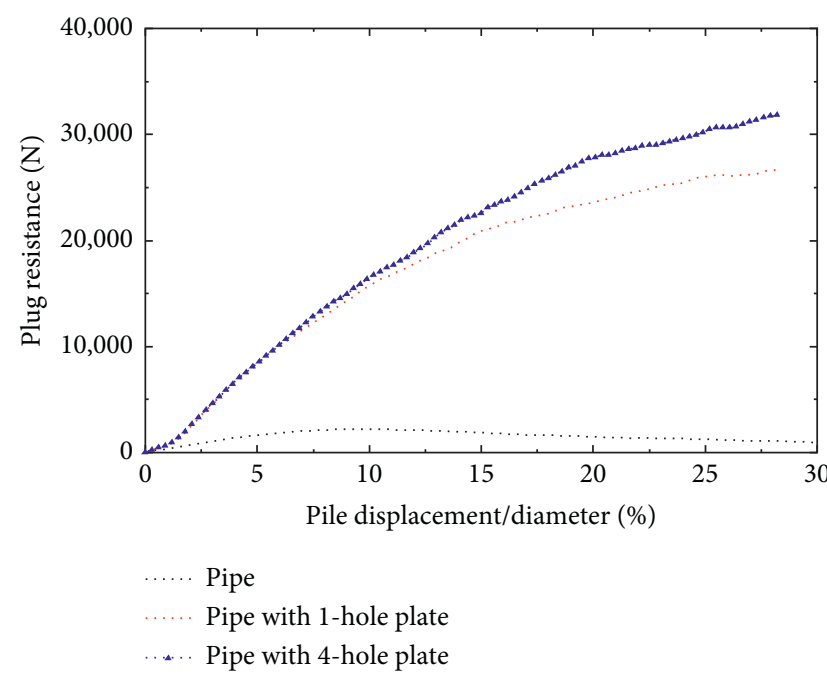

(a)

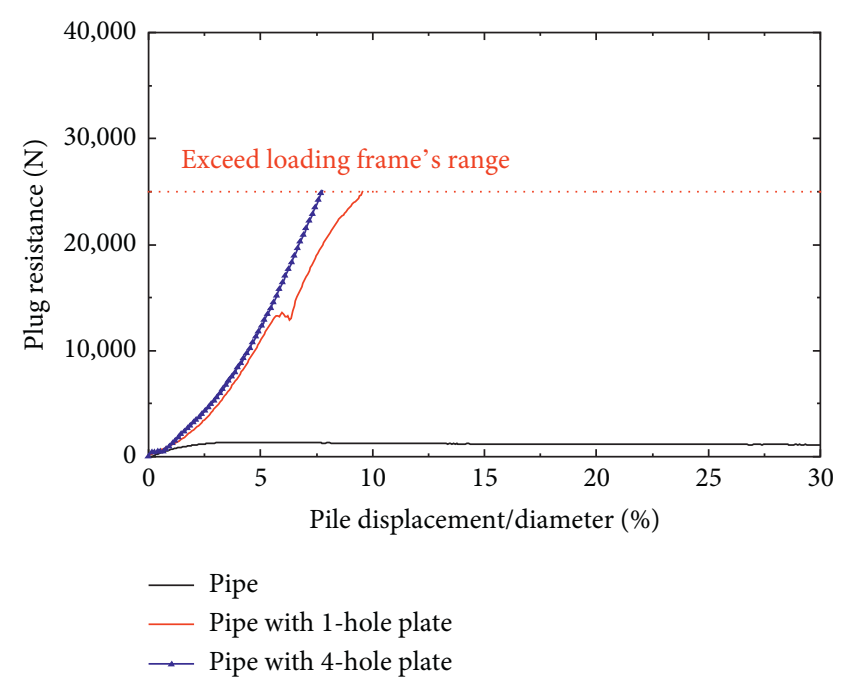

(b)

Figure 9: Axial force-displacement curves of pipe piles with different restriction plates: (a) simulation results and (b) experimental results.

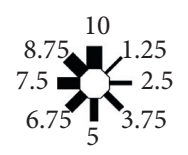

Unit: N

Displacement /diameter:

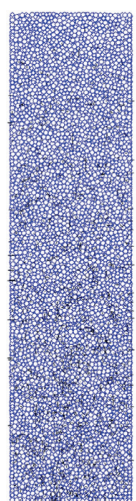

$0 \%$

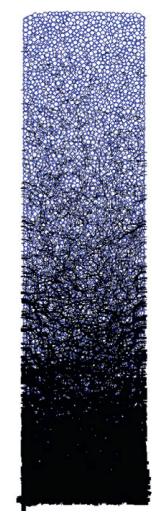

$10 \%$

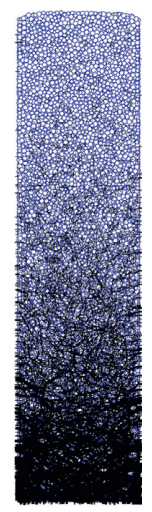

$20 \%$

Figure 10: Particle contact force transmission in a pipe pile $(D=10 \mathrm{~cm}$ and $L / D=4.0)$.

as the DEM model only solves for discrete particle interactions; however, the particle stress provides comparable results regarding the compaction state of the granular materials. As shown in Figure 14, the average contact stress increases dramatically with the incorporation of the restriction plate, which corresponds to the significant increase in the soil plug resistance. It is also shown that the contact stress increases almost linearly from top to bottom even with the restriction plate, and soil particles at the bottom part are subjected to the highest confinement:

$$
\sigma_{c}=\frac{F_{c, \max }}{\pi R^{2}},
$$

where $F_{c, \max }$ is the maximum contact force; $R$ is the particle radius; and $\sigma_{c}$ is the defined particle stress.

Figure 15 compares the soil plug resistance of the DEM models with one-hole restriction plates of different-sized circular holes. The plug resistance has been normalized by dividing by the resistance of the open-ended pipe pile, and the normalized plug resistance equals 1.0 for the pipe pile without a restriction plate. It is shown that the normalized plug resistance increases exponentially with the decrease of the plate inner circular hole. A mathematical regression is also provided in Figure 15, which could help to estimate the plug resistance with different plate designs. It is shown that, by properly adjusting the inner hole diameter, engineers could optimize the restriction plate design based on the end bearing capacity and the pile drivability.

4.3. Centrifuge Scale up of Numerical Model. The extremely high computational cost prohibits the implementation of the DEM simulation for large-diameter open-ended pipe piles. Here, we propose a scaleup scheme by applying the geotechnical centrifuge principle into the DEM model. The centrifuge experiment tests small-scale models under high gravitational levels to emulate the stress state of field conditions. By applying a higher gravitational acceleration, the geometric dimension of the numerical model is 

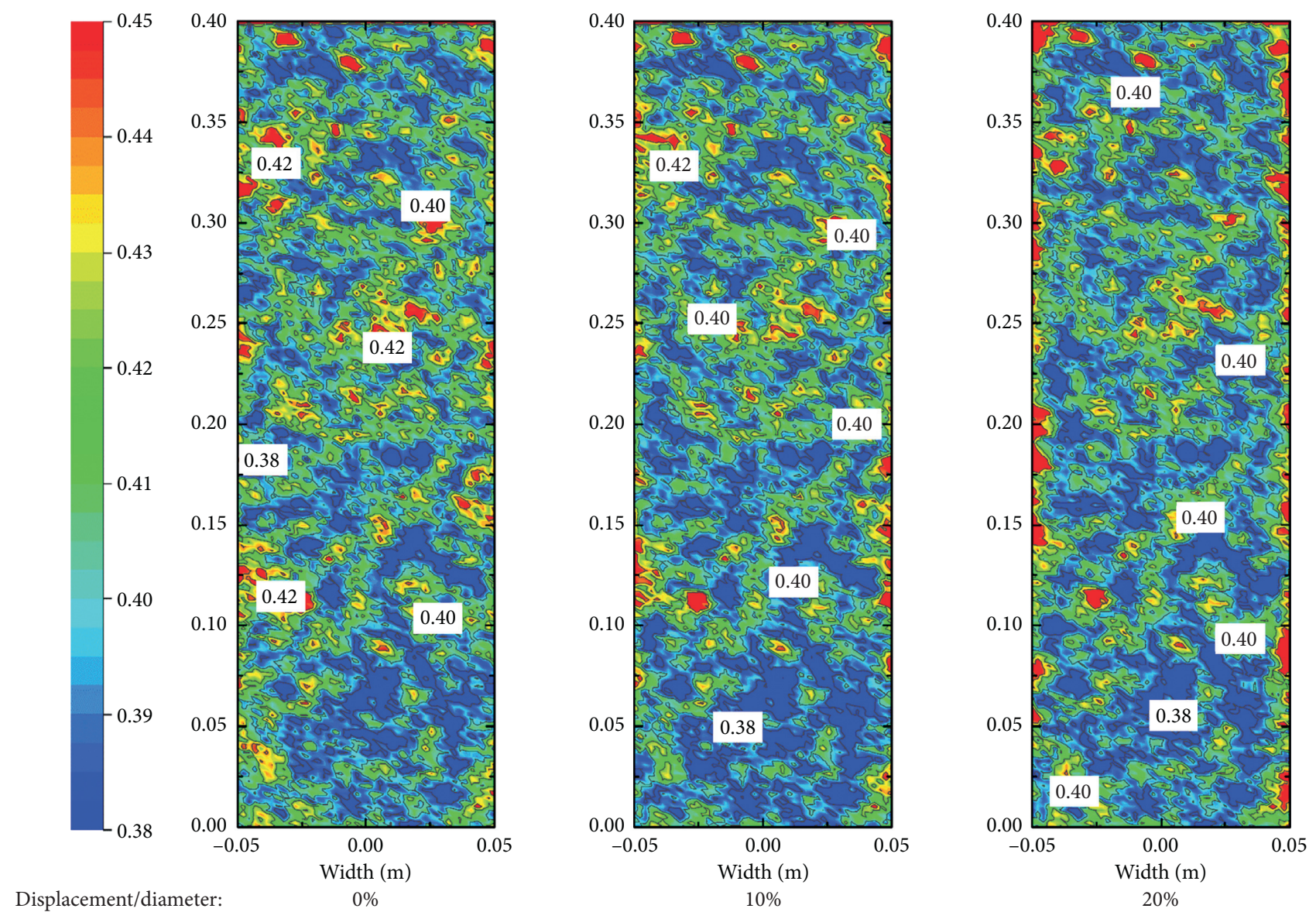

FIgURE 11: Porosity distribution inside the soil plug $(D=10 \mathrm{~cm}$ and $L / D=4.0)$.

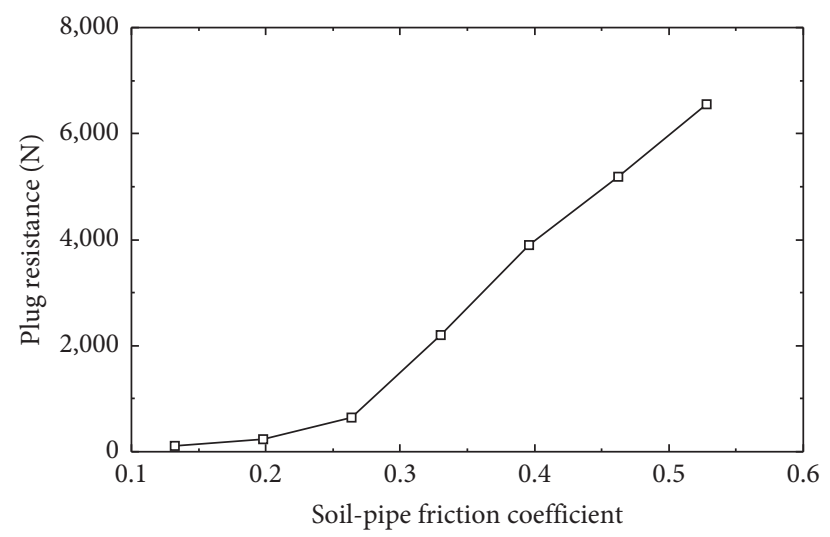

Figure 12: Soil plug resistance of numerical models with different soil-pile friction coefficients $(D=10 \mathrm{~cm}$ and $L / D=4.0)$.

scaled up based on the scale factors, as shown in Table 4 [43]. Following this principle, the DEM model is scaled up to analyze the plug resistance of large diameters. A constant length-diameter ratio of 4.0 is maintained for all the model piles in this study. Model contact parameters are the same as listed in Table 1, as the material properties (e.g., Young's modulus, internal friction angle, and density) should be the same between prototypes and models.
The simulation result of the plug unit area resistance of different pipe piles is given in Figure 16, in which the unit area resistance equals the plug resistance divided by the cross section area. From Figure 16, the pipe pile with four-hole restriction plate tends to form a higher degree of soil plugging compared to that with one-hole restriction plate, both of which provide a much higher resistance than the pipe pile. With the increase of the pile diameter, the unit area resistance decreases as the arching effect inside the soil 


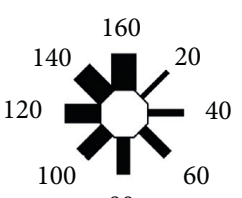

80

Unit: N

1-hole restriction plate

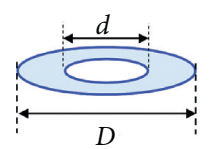

4-hole restriction plate

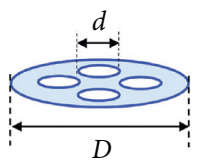

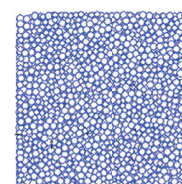
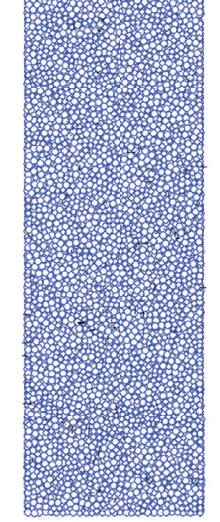

Before test

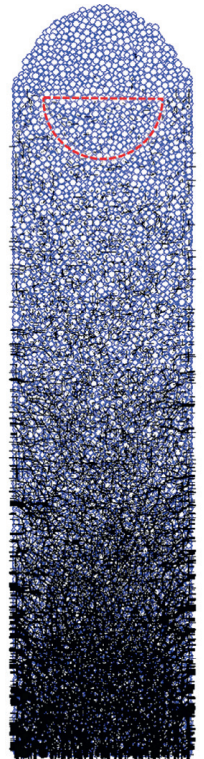

1-hole plate, $d / D=0.75$

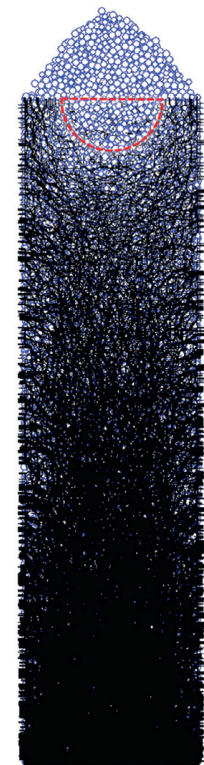

1-hole plate, $d / D=0.50$

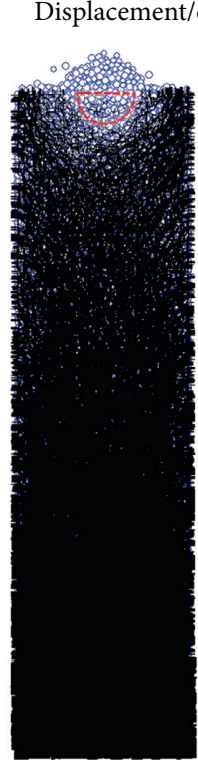

1-hole plate, $d / D=0.25$

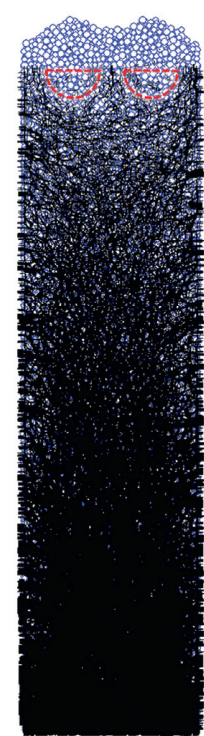

4-hole plate, $d / D=0.25$

FIGURE 13: Particle contact force transmission inside soil plugs with different types of restriction plates $(D=10 \mathrm{~cm}$ and $L / D=4.0)$.

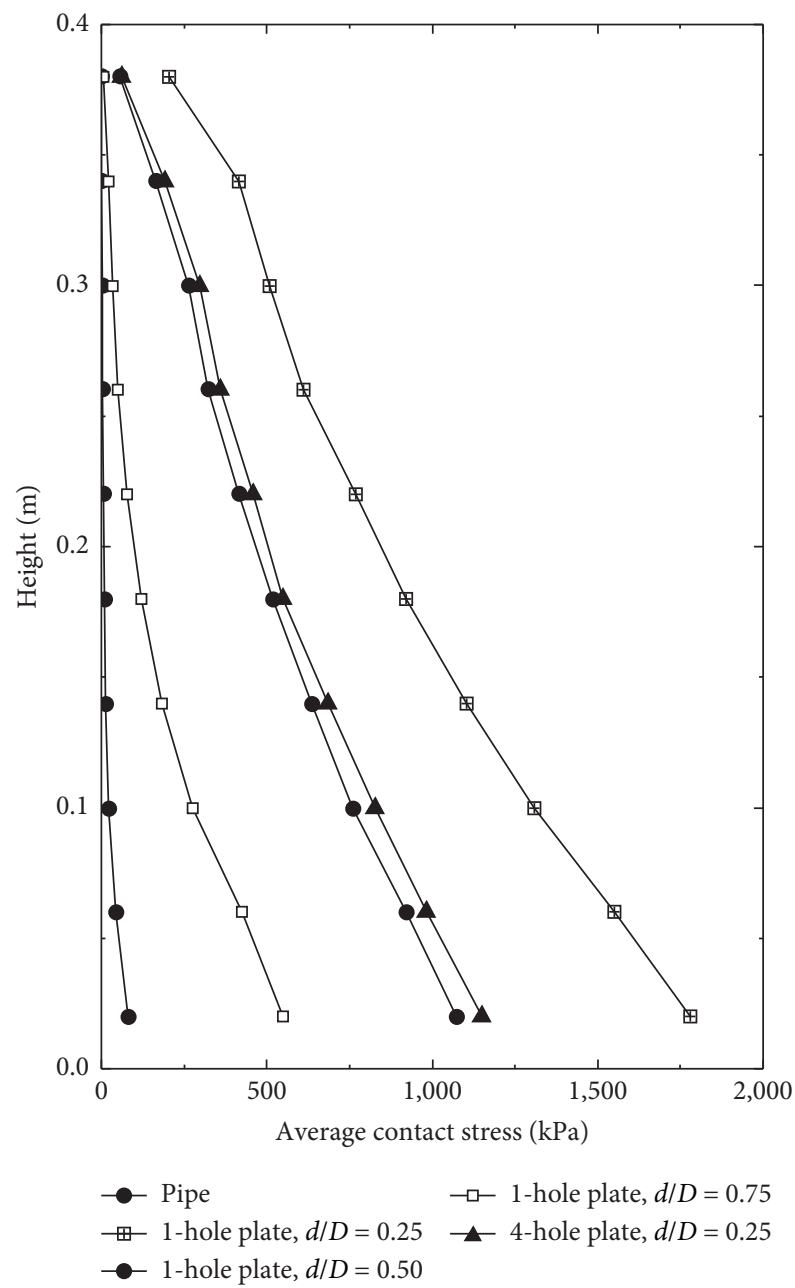

Figure 14: Average particle contact stress inside the soil plug over heights $(D=10 \mathrm{~cm}$ and $L / D=4.0)$. 


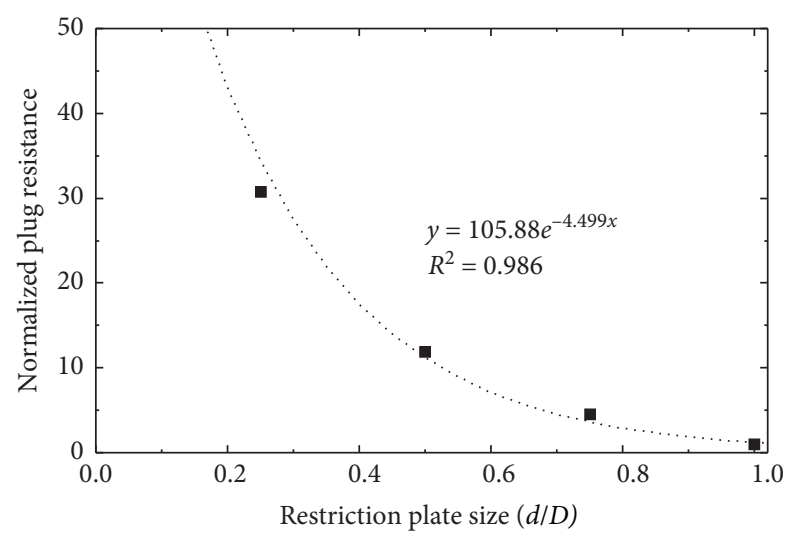

FIgURE 15: Normalized soil plug resistance with one-hole restriction plates of different inner hole diameters $(D=10 \mathrm{~cm}$ and $L /$ $D=4.0)$.

TABle 4: Scale factors for geotechnical centrifuge modeling.

\begin{tabular}{lcc}
\hline Quantity & Units & Scale factor \\
\hline Length & $\mathrm{L}$ & $1 / N$ \\
Volume & $\mathrm{L}^{3}$ & $1 / N^{3}$ \\
Mass & $\mathrm{M}$ & $1 / N^{3}$ \\
Gravity & $\mathrm{L} / \mathrm{T}^{2}$ & $N$ \\
Force & $\mathrm{ML} / \mathrm{T}^{2}$ & $1 / N^{2}$ \\
Stress & $\mathrm{M} / \mathrm{LT}^{2}$ & 1 \\
Moduli & $\mathrm{M} / \mathrm{LT}^{2}$ & 1 \\
Strength & $\mathrm{M} / \mathrm{LT}^{2}$ & 1 \\
\hline
\end{tabular}

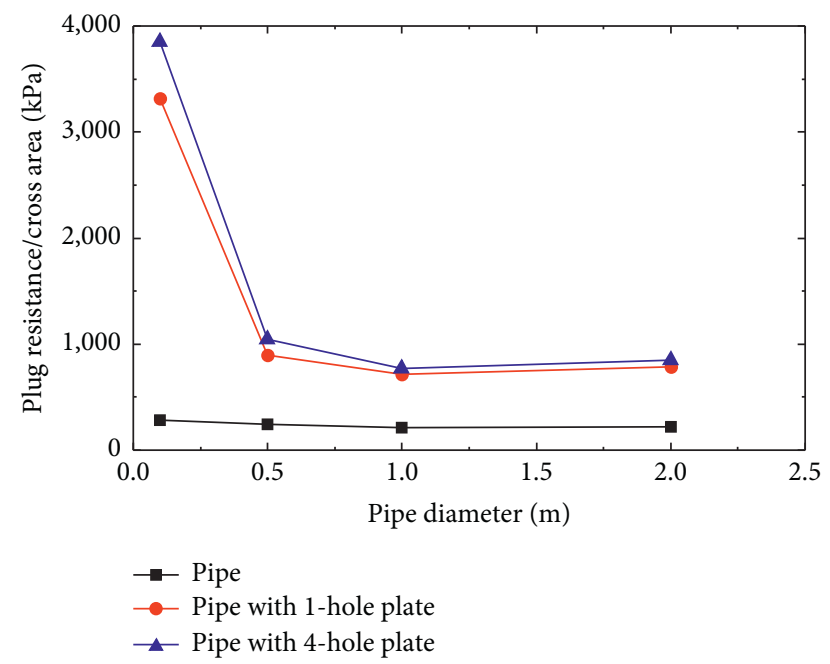

FIgURE 16: Unit area plug resistance of pipe piles of different diameters with/without restriction plates from geotechnical centrifuge simulations $(L / D=4.0)$.

column is weakened. A significant drop is observed for the piles with restriction plates as the pile diameter increases from $0.1 \mathrm{~m}$ to $0.5 \mathrm{~m}$. Above $1.0 \mathrm{~m}$, the unit area resistance becomes relatively stable. However, even for the $2 \mathrm{~m}$ in diameter pipe piles, the plug resistance is increased by $252 \%$ with one-hole restriction plate and $281 \%$ with four-hole restriction plate. Overall, large-diameter pipe piles are more difficult to form a soil plug at the same length-diameter ratio. The use of restriction plates is effective to force the soil plug to form and increases the equivalent end bearing capacity compared to traditional open-ended pipe piles, including at large pile diameters.

\section{Summary and Conclusions}

For large-diameter open-ended pipe piles, the end bearing capacity depends highly on the soil plugging behavior inside the pipe wall. This paper studies the soil plugging mechanism inside the open-ended pipe piles by use of the computational simulations and the laboratory experiments. A DEM model of the soil plug is built to analyze the soil plug resistance and is verified by comparison to the results from the laboratory experiments. Two innovatively designed restriction plates, that is, a one-hole restriction plate and a four-hole restriction plate, are proposed, which are installed inside the pipe wall to increase the soil plug resistance and achieve desirable results.

It is shown that the frictional force transmission among soil particles is the key in the formation of a soil plug. Under a specified soil condition, the friction coefficient of soil-pile interface, the pile diameter, and the plug length-diameter ratio are three important factors determining the plugging behavior of the pipe piles. Both the laboratory experiment and the numerical simulation indicate that the use of restriction plates significantly improves the soil plug resistance. The four-hole restriction plate tends to be more effective than one-hole restriction plate. Furthermore, the geotechnical centrifuge principle has been adopted by the DEM model to scale up the model dimensions for analyzing the plugging behavior inside the large-diameter pipe piles. The results show that, with the increase of the pile diameter, the plug's unit area resistance and the degree of soil plugging are generally decreased. However, large-diameter pipe piles with restriction plates still achieve significantly higher unit area resistance than the traditional pipe piles without a restriction plate.

\section{Data Availability}

The experimental data used to support the findings of this study are included within the article.

\section{Additional Points}

Highlights. (i) Combined experimental and numerical study on soil plug resistance. (ii) Two innovatively designed restriction plates to facilitate the soil plug formation. (iii) Visualization of the contact force concentration in numerical models. (iv) Geotechnical centrifuge simulation to scale up model dimensions.

\section{Conflicts of Interest}

The authors declare that there are no conflicts of interest regarding the publication of this paper. 


\section{Acknowledgments}

The research project was supported by the Ohio Department of Transportation (ODOT) under Grant \#135103. The technical assistance and financial support from this organization are highly appreciated.

\section{References}

[1] NCHRP Synthesis 478, Design and Load Testing of Large Diameter Open-Ended Driven Piles, Transportation Research Board, Washington, DC, USA, 2015.

[2] R. F. Stevens, E. A. Wiltsie, and T. H. Turton, "Evaluating pile drivability for hard clay, very dense sand, and rock," in Proceedings of the Fourteenth Annual Offshore Technology Conference, vol. 1, pp. 465-481, Houston, TX, USA, 1982.

[3] L. Sa, T. Yinghui, Z. Yangrui, J. Baofan, and W. Jinbiao, "Premature refusal of large-diameter, deep-penetration piles on an offshore platform," Applied Ocean Research, vol. 42, pp. 55-59, 2013.

[4] S. Mohan, R. Stevens, R. Howard, and M. Kapuskar, "Design of large diameter driven pipe pile foundations: New east span san francisco-oakland bay bridge," in Proceedings of the Geotechnical Engineering for Transportation Projects, pp. 278-285, Los Angeles, CA, USA, July 2004.

[5] B. A. Liebich, "High-capacity piles at the stony creek bridge project," Transportation Research Record: Journal of the Transportation Research Board, vol. 2116, no. 1, pp. 41-46, 2009.

[6] M. F. Randolph, R. Dolwin, and R. Beck, "Design of driven piles in sand," Géotechnique, vol. 44, no. 3, pp. 427-448, 1994.

[7] K. Paik, R. Salgado, J. Lee, and B. Kim, "Behavior of open- and closed-ended piles driven into sands," Journal of Geotechnical and Geoenvironmental Engineering, vol. 129, no. 4, pp. 296306, 2003.

[8] J. Dijkstra and W. Broere, "Experimental investigation into plugging of open ended piles," in Proceedings of the ASME 2009 28th International Conference on Ocean, Offshore and Arctic Engineering, pp. 1-8, Honolulu, HI, USA, January 2009.

[9] M. W. O’Neill and R. D. Raines, "Load transfer for pipe piles in highly pressured dense sand," Journal of Geotechnical Engineering, vol. 117, no. 8, pp. 1208-1226, 1991.

[10] A. S. Bradshaw, S. Haffke, and C. Baxter, "Load transfer curves from a large-diameter pipe pile in silty soil," in Proceedings of the Full-Scale Testing and Foundation Design, pp. 590-601, Oakland, CA, USA, March 2012.

[11] H. Kishida and N. Isemoto, "Behavior of sand plugs in openend steel pipe piles," in Proceedings of the 9th International Society for Soil Mechanics and Foundation Engineering, pp. 601-604, Tokyo, Japan, 1977.

[12] P. J. Hannigan, G. G. Goble, G. E. Likins, and F. Rausche, Design and Construction of Driven Pile Foundations, Federal Highway Administration, Washington, DC, USA, 2006.

[13] American Petroleum Institute, "Geotechnical and foundation design considerations," American Petroleum Institute, Washington, DC, USA, 2011.

[14] G. A. Miller and A. J. Lutenegger, "Influence of pile plugging on skin friction in overconsolidated clay," Journal of Geotechnical and Geoenvironmental Engineering, vol. 123, no. 6, pp. 525-533, 1997.

[15] D. S. Liyanapathirana, A. J. Deeks, and M. F. Randolph, "Numerical analysis of soil plug behaviour inside open-ended piles during driving," International Journal for Numerical and
Analytical Methods in Geomechanics, vol. 22, no. 4, pp. 303-322, 1998.

[16] K. G. Gavin and B. M. Lehane, "The shaft capacity of pipe piles in sand," Canadian Geotechnical Journal, vol. 40, no. 1, pp. 36-45, 2003.

[17] Y. Choi, M. S. Nam, and M. W. O’Neill, "Response of openended piles in sand to simulated earthquake and seaquake," International Journal of Offshore and Polar Engineering, vol. 10, no. 3, pp. 229-235, 2000.

[18] H. Liu, W. Wu, G. Jiang, M. H. El Naggar, G. Mei, and R. Liang, "Influence of soil plug effect on the vertical dynamic response of large diameter pipe piles," Ocean Engineering, vol. 157, pp. 13-25, 2018.

[19] K. Paik and R. Salgado, "Determination of bearing capacity of open-ended piles in sand," Journal of Geotechnical and Geoenvironmental Engineering, vol. 129, no. 1, pp. 46-57, 2003.

[20] B. M. Lehane and K. G. Gavin, "Base resistance of jacked pipe piles in sand," Journal of Geotechnical and Geoenvironmental Engineering, vol. 127, no. 6, pp. 473-480, 2001.

[21] J. A. Schneider, X. Xu, and B. M. Lehane, "End bearing formulation for CPT based driven pile," in Proceedings of the CPT'10 2nd International Symposium on Cone Penetration Testing, vol. 3, pp. 1-8, Hundtington Beach, CA, USA, 2010.

[22] D. J. P. Igoe, K. G. Gavin, and B. C. O’Kelly, "Shaft capacity of open-ended piles in sand," Journal of Geotechnical and Geoenvironmental Engineering, vol. 137, no. 10, pp. 903-913, 2011.

[23] S. Jeong, J. Ko, J. Won, and K. Lee, "Bearing capacity analysis of open-ended piles considering the degree of soil plugging," Soils and Foundations, vol. 55, no. 5, pp. 1001-1014, 2015.

[24] Y. Guo and X. B. Yu, "Design and analyses of open-ended pipe piles in cohesionless soils," Frontiers of Structural and Civil Engineering, vol. 10, no. 1, pp. 22-29, 2016.

[25] B. M. Lehane, J. A. Schneider, and X. Xu, "A review of design methods for offshore driven piles in siliceous sand," UWA Report No. GEO 05358, The University of Western Australia, Perth, Australia, 2005.

[26] R. J. Jardine, F. C. Chow, R. Overy, and J. Standing, ICP Design Methods for Driven Piles in Sands and Clays, Thomas Telford, London, UK, 2005.

[27] F. Yu and J. Yang, "Base capacity of open-ended steel pipe piles in sand," Journal of Geotechnical and Geoenvironmental Engineering, vol. 138, no. 9, pp. 1116-1128, 2012.

[28] S. R. Gudavalli, O. Safaqah, and H. Seo, "Effect of soil plugging on axial capacity of open-ended pipe piles in sands," in Proceedings of the 18th International Conference on Soil Mechanics and Geotechnical Engineering, pp. 1487-1490, Paris, France, 2013.

[29] M. Y. Abu-Farsakh, M. N. Haque, and C. Tsai, "A full-scale field study for performance evaluation of axially loaded largediameter cylinder piles with pipe piles and PSC piles," Acta Geotechnica, vol. 12, no. 4, pp. 753-772, 2017.

[30] M. F. Randolph, E. C. Leong, and G. T. Houlsby, "One-dimensional analysis of soil plugs in pipe piles," Géotechnique, vol. 41, no. 4, pp. 587-598, 1991.

[31] C. Zheng, H. Liu, and X. Ding, "Lateral dynamic response of a pipe pile in saturated soil layer," International Journal for Numerical and Analytical Methods in Geomechanics, vol. 40, no. 2, pp. 159-184, 2016.

[32] H. Peiffer, "Predicting the settlement behaviour of openended pipe piles with a large diameter," Géotechnique Letters, vol. 7, no. 3, pp. 225-229, 2017.

[33] Z. Li, K. Wang, W. Wu, C. J. Leo, and N. Wang, "Vertical vibration of a large-diameter pipe pile considering the radial 
inhomogeneity of soil caused by the construction disturbance effect," Computers and Geotechnics, vol. 85, pp. 90-102, 2017.

[34] J. Ko, S. Jeong, and J. K. Lee, "Large deformation FE analysis of driven steel pipe piles with soil plugging," Computers and Geotechnics, vol. 71, pp. 82-97, 2016.

[35] M. Zhou, H. Liu, M. S. Hossain, Y. Hu, and T. Zhang, "Numerical simulation of plug formation during casing installation of cast-in-place concrete pipe (PCC) piles," Canadian Geotechnical Journal, vol. 53, no. 7, pp. 1093-1109, 2016.

[36] P. A. Cundall and O. D. L. Strack, "A discrete numerical model for granular assemblies," Géotechnique, vol. 29, no. 1, pp. 47-65, 1979.

[37] Y. Guo and X. Yu, "Soil plugging mechanism on large diameter pipe piles: insight from discrete element simulations," in Proceedings of the IFCEE 2015, San Antonio, TX, USA, March 2015.

[38] X. Zhang, B. Fatahi, H. Khabbaz, and B. Poon, "Assessment of the internal shaft friction of tubular piles in jointed weak rock using the discrete-element method," Journal of Performance of Constructed Facilities, vol. 33, no. 6, Article ID 04019067, 2019.

[39] Itasca Consulting Group, "Particle flow code in 3 dimensions (PFC3D) manual,” Itasca Consulting Group, Minneapolis, MN, USA, 2014.

[40] ASTM International, Standard Test Methods for Specific Gravity of Soil Solids by Water Pycnometer, ASTM International, West Conshohocken, PA, USA, 2002.

[41] ASTM International, Standard Test Methods for Maximum Index Density and Unit Weight of Soils Using a Vibratory Table, ASTM International, West Conshohocken, PA, USA, 2016.

[42] ASTM International, Standard Test Methods for Minimum Index Density and Unit Weight of Soils and Calculation of Relative Density, ASTM International, West Conshohocken, PA, USA, 2016.

[43] S. Iai, T. Tobita, and T. Nakahara, "Generalised scaling relations for dynamic centrifuge tests," Géotechnique, vol. 55, no. 5, pp. 355-362, 2005. 